\title{
Mixed Tumor, Not Otherwise Specified
}

National Cancer Institute

\section{Source}

National Cancer Institute. Mixed Tumor, Not Otherwise Specified. NCI Thesaurus. Code C121786.

A benign myoepithelioma characterized by the presence of a minor ductal component. 